\title{
IL BATISISMO DEL 28 AGOSTO 1946 E LA PROFONDITA' DEL NUCLEO TERRESTRE
}

\author{
P. Calor - F. Peronaci
}

If 28 agosto 1946 venne registrato a Roma un terremoto con le caratteristiche proprie dei movimenti originanti a grandissima profondità. Pensammo di farne oggetto di particolare indagine. A tale scopo richiedemmo, ai vari Osservatori sparsi per il mondo, gli originali io copie fotografiche) dei sismogrammi da esso destati. L'intensità del terremoto non fu notevole, le difficolta inerenti allo stato di guerra, da poco cessata, ancora in parte esistenti: il numero delle recristrazioni ottenute, pertanto, non fu grande. Comunque, potemmo avere a disposizione i sismogrammi di una ventina di Osservatori.

1. Epicentro, profondità ipocentrale. - Nostra prima preoccupazione fu la determinazione delle coordinate ipocentrali.

Con i valori ottenuti da Pasadena $l_{\varphi}=26^{\circ} \mathrm{S}$; $i=63^{\circ} \mathrm{W}$;

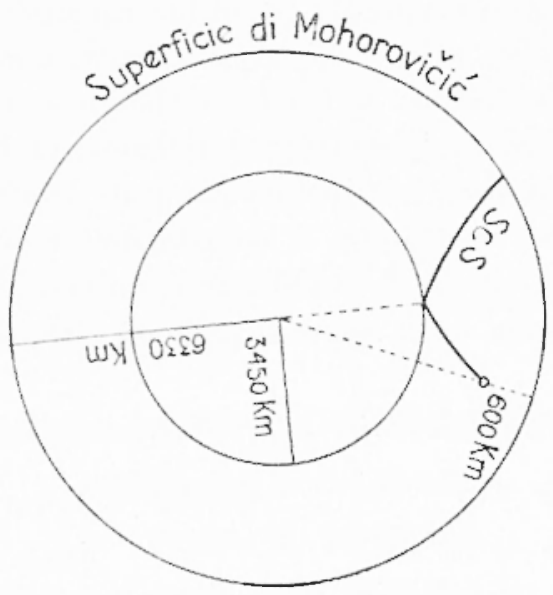

Fig. 1

$h=580 \mathrm{~km}$, procedemmo ad una prina approssimazione per le coordinate epicentrali isupposto $h=580 \mathrm{~km}$ ) con un metodo esposto in un nostro precedente lavoro (') I dati di cui ci siamo serviti sono 
quelli riportati nella tabella I; i risultati ollenuti confermarono sostanzialnente quelli indicali da Pasalena, con un'ora origine pari a

$$
H=28^{\mathrm{m}} \quad 23^{*}: 0
$$

Siano quindi passati alla determinazione delle grandezze ricercate, considerando incognita anche la profondita. II metodo scguito quello da noi esposto nel lavoro citalo ('), grià applicato con successo anche da altri ricercatori. Esso si compendia essenzialmente nella soluzione dell'equazione

$$
\begin{aligned}
& \partial t_{0}-\cos u_{0}^{\prime} \sin u \frac{\partial f}{\partial J_{11}} \cdot \partial \partial_{0}-\cos u \frac{\partial f}{\partial J_{11}} \cdot 0 u \omega_{0}+\frac{\partial f}{\partial h_{0}} \cdot \sigma h_{0} \\
& =\left(P_{\mathrm{n}}\right)-t_{0}-f\left(\lambda_{0}, \varphi^{\prime}{ }_{0}, h_{0}\right),
\end{aligned}
$$

dove, fra gli altri simboli nunifesti, $t_{0}$ i il lempo origine approssimato e $\delta t_{0}$ la relativa correzione, u l'azimul dell'epicentro rispetto a ciascuna stazione, $\left(P_{n}\right)$ indica i tempi di registrazione lelle onde $P$ nelle stazioni di osservazione, $f\left(\lambda_{0}, \varphi_{o}, h_{o}\right)$ i il tempo di propayazione delle $P$ per la slazione considerala, in hase ai valori approssimali di $\iota_{a}, \varphi_{o} ; \partial f, \partial h_{0}$ si ottiene dalle dromocrone, fatto $\Delta$ costante nel valore $J_{0}$ ed assegnando ad $h$ (a partire da $\left.h_{n j}\right)$ un increnento pari all'unita della dromocrona prescelta, mentre $\partial f / \partial J_{n}$ si calcola, sempre dalle dromocrone, supponendo costante $h$ (in $h_{0}$ ) e dando a $J_{n}$ l'increntento di $1^{\circ}$ per avere quello corrispondente del tempo di tragitto.

La [1] naturalnente va ripetuta per tutle le stazioni e il sistema che così si ottiene, risolto con il metodo dei minimi quatrati. Con i dali forniti dalla tabella I e calcolando con coordinate ereocentriche si sono ottenuti (alla terza approssimazione) i seguenti risultati:

$$
\begin{aligned}
& r^{\prime}=24^{\prime \prime} 47^{\prime}, 25,34^{\prime} \text { (geocentrica) } \\
& \tau_{\circ}=24^{\circ} 56^{\prime}, 05 \mathrm{~S} \text { (geografica) } \\
& \lambda_{0}=62^{\circ} 59^{\prime}, 95 W^{\prime}+6^{\prime} \\
& \boldsymbol{h}=643 \mathrm{Km} .+\frac{-1 .}{29} \mathrm{Km} \text {. } \\
& t_{\mathrm{o}}=28^{\mathrm{m}} 32^{\mathrm{s}}, 8 \cdots 4^{\mathrm{n}}, 2 \text {. }
\end{aligned}
$$

La bontà dei calcoli d data dalla pratica coincidenza di [II. 4] con $[\mathrm{v}]$; si è avuto infatti $|11.4|=7,022277,[\mathrm{vv}]=7,022275$. 


\section{Tabelat I}

\begin{tabular}{|c|c|c|c|}
\hline Stizioni & \multicolumn{3}{|c|}{ Tempi osservalli per le onde $P$} \\
\hline Huancayo & 22 & $\begin{array}{r}m \\
32\end{array}$ & 2,8 \\
\hline Chicago & " & 38 & 44,8 \\
\hline Tucson A. & 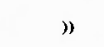 & 38 & 59,3 \\
\hline Lal Jolla & $"$ & 39 & 25,0 \\
\hline Monte Wilson & $"$ & 39 & 29,5 \\
\hline Pasadena & $"$ & 39 & 30.3 \\
\hline S. Barbara & 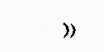 & 39 & 37,3 \\
\hline Haiwee & $"$ & 39 & 37,6 \\
\hline Tinemaha & $n$ & 39 & 41,8 \\
\hline Kew & $"$ & 40 & 42,8 \\
\hline Zurigro & 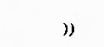 & 40 & 56,0 \\
\hline Roma & $"$ & 40 & 55,3 \\
\hline
\end{tabular}

Tabelala II

\begin{tabular}{|l|c|l|c|}
\hline \multicolumn{1}{|c|}{ Stizioni } & Distanze in gradi & Stizioni & Distanze in gradi \\
\hline Montezuma & 10,18 & Iaiwee & 79,93 \\
La Plata & 11,12 & Tinemaha & 80,53 \\
Huancayo & 17,32 & Toledo & 91,26 \\
S. Juan & 43,05 & Berkeley & 92,58 \\
Chicago & 70,17 & Kew & 93,70 \\
Tucson A. & 72,72 & Zurigo & 96,06 \\
La Jolla & 77,22 & Roma & 96,15 \\
Monte Wilson & 78,59 & Coira & 96,67 \\
Pasadena & 78,61 & Sitka & 101,44 \\
\end{tabular}


Nella tabella II sono contenute le distanze epicentrali di 18 stazioni (i dati di aleune delle quali ci sono pervenuti a calcoli ullimati).

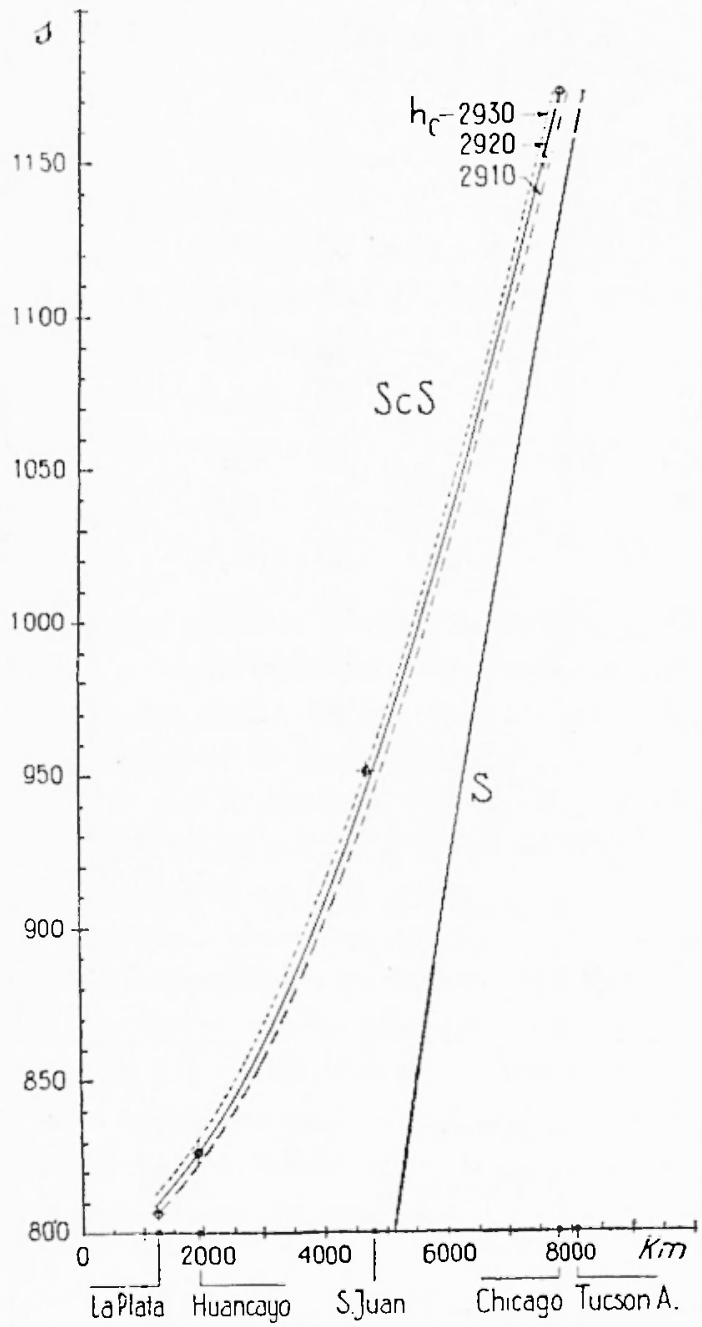

Fig. 2
2. Profonditía del nucleo centrule della Terra. - Il terremoto in esame ha provocato lat registrazione di alcuni nellissimi esempi di onde $S c S$ ( fig. 4). Ritenemmo utile valerci dei relativi tempi di arrivo per risalire, noti i valori delle altre grandezze in grioco, alla profonditá del nucleo centrale terrestre.

Nella firr. 1 i schematizzato il tragitlo delle onde SaS. 11 ragrgrio $r "$ si riferisce alla discontinuita di Mohorovic.

Se $r$ ì il ragrgio di un punto grenerico di una traieltoria sismical, si sal che il tempo di tragitto $d t$ relativo ad un elemento di traiet. toria $\dot{c}$ dato da

$$
d t=\frac{n^{2} r d r}{\sqrt{n^{2} r^{2}-l^{2}}}
$$

dove $n=\frac{1}{v}$, essendo $v$ la velocità nel punto generico considerato, $\mathrm{e}$ $k=n r \cos e$, rappresentando e l'angolo d'emergenza nel punto.

L'indice o riferisec le grandezze alla superficie di Mohorovicic, l'indice 1 al punto corrispondente alla profondità ipocentrale e l'in- 
dice 2 al nucleo centrale; pertanto, avemo per il tempo di tragito di un'onda $S c S$ :

$$
T=n_{0} r_{0}\left(\int_{Q_{2}}^{\rho_{1}} \frac{v^{2} d_{0}}{\sqrt{q^{2}-u_{0}^{2}}}+\int_{Q_{2}}^{e_{0}} \frac{v_{0} d_{0}}{\sqrt{q^{2}-\alpha^{2}}}\right),
$$

dovi: :

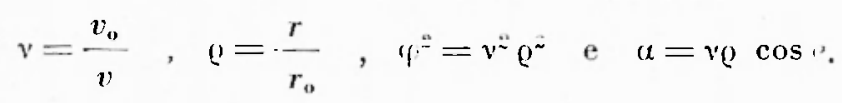

Occorreva, a questo punto, trovare una legge di distribuzione della velocità delle onde trasversali (S) nel " mantello " terrestre, che consentisse un'opportuna espressione di 0 in funzione di q $^{*}$. Partendo dai valori per la velocita delle onde $S$ nel mantello, dati da Gutenherg e Richter (*) si è trovalo - - conformemente ai risultati di analoghe ricerche - che, con buonissima approssimazione, si ha:

$$
0=\frac{r^{2}}{c+C_{4}^{2}}
$$

dove $c$, C sono costanti, il cui valore si ì potuto ottenere dalla soluzione, con il metodo dei minimi quadrati, di 21 equazioni, corrispondenti a 21 valori della velocita delle $S$ a diverse profondita ipocentrali, da quota 10 (discontinutí di Mohorovicic) a quota 2920. Ne is risultato

$$
r=0.09228 \quad, \quad C=0.90325 .
$$

Per la [2]. la [3] diviene

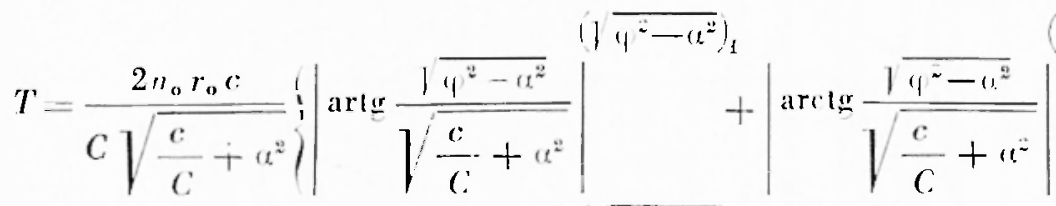

$$
\begin{aligned}
& \left(\sqrt{y^{2}-a^{2}}\right)_{2} \\
& \left(\sqrt{4^{2}-10^{2}}\right)_{0} \\
& \left(\sqrt{y^{2}--13^{2}}\right)
\end{aligned}
$$

Il valore di a per le singole stazioni fu calcolato usufruendo dei valori di $\sin i_{2}$ riportati nel lavoro cilato di Gutenheres e Richter (pagina 10\% in cui $\theta$, va corretto tenendo debito conto della profondita fcio che si i fatto ricorrendo ad altro lavoro di Gutenbere e Richter) (").

Eceo i risultati dei calcoli ottenuti con la [4] (ritenendo pari a 13 sec in media il tempo di tragitto nella crosta terrestre) 

Tempo di trit- Tragitlo per
gitlo Sis os- per profon-
servito

$\begin{array}{lccccccc}\text { Huancayo } & 1908 \mathrm{~km} & 13^{\mathrm{m}} & 59^{\circ} & 13^{\mathrm{m}} & 59^{\circ} & 2920 & \mathrm{~km} \\ \text { La Plata } & 1234 \mathrm{~km} & 13 & 1371 & 13 & 40 & (2905) \mathrm{km} \\ \text { San Juan } & 1780 \mathrm{~km} & 16 & 1 & 16 & 03 & 2930 & \mathrm{~km} \\ \text { Chicago } & 7800 \mathrm{~km} & 19 & 21 & 19 & 21 & 2920 & \mathrm{~km} \\ \text { Tucson } & 8080 \mathrm{~km} & 19 & 11 & 19 & 19 & 2920 & \mathrm{~km}\end{array}$

Possiamo quindi ritenere senzaluro come profondita del nucleo

$$
h_{x}=2920 \mathrm{~km} \text {, }
$$

valore che coincide con quello oltenuto da Gutenlerer con il metodo di Wiechert-Iterglotz.

È noto che Dahm ( ${ }^{4}$ ) in una ricerca condolla pure con il melodo di Wiechert-Herglotz, ì giunto alla conchusione che verso $2700 \mathrm{~km} \mathrm{di}$ profondita la velocita delle onde longitudinali subisce una diminuzione, che si mantiene fino a $3000 \mathrm{~km}$, profondita alla quale avrebbe inizio il nucleo. Ci siamo proposti di vedere a quali risultati conduce la $[4]$, determinando le costanti $c, C$ che figurano nella $[3]$ con le

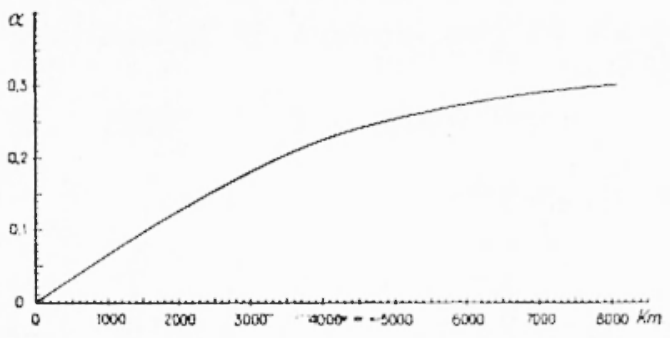

Fig. 3

velocita ottenute da Dahm nel suo lavoro per la velocita delle onde $S$ nel mantello.

Risolte, con il melodo dei minimi quadrati, le 54 equazioni in $c, C$ che risultano in corrispontenza dei valori della velocita alle varie quote (da $40 \mathrm{~km}$ a $2700 \mathrm{~km}$ ), si ì ottenuto

$$
c=0,103215 \quad, \quad C=0,906136
$$

Per l'applicazione della [4] restavano da determinare $i$ valori di 


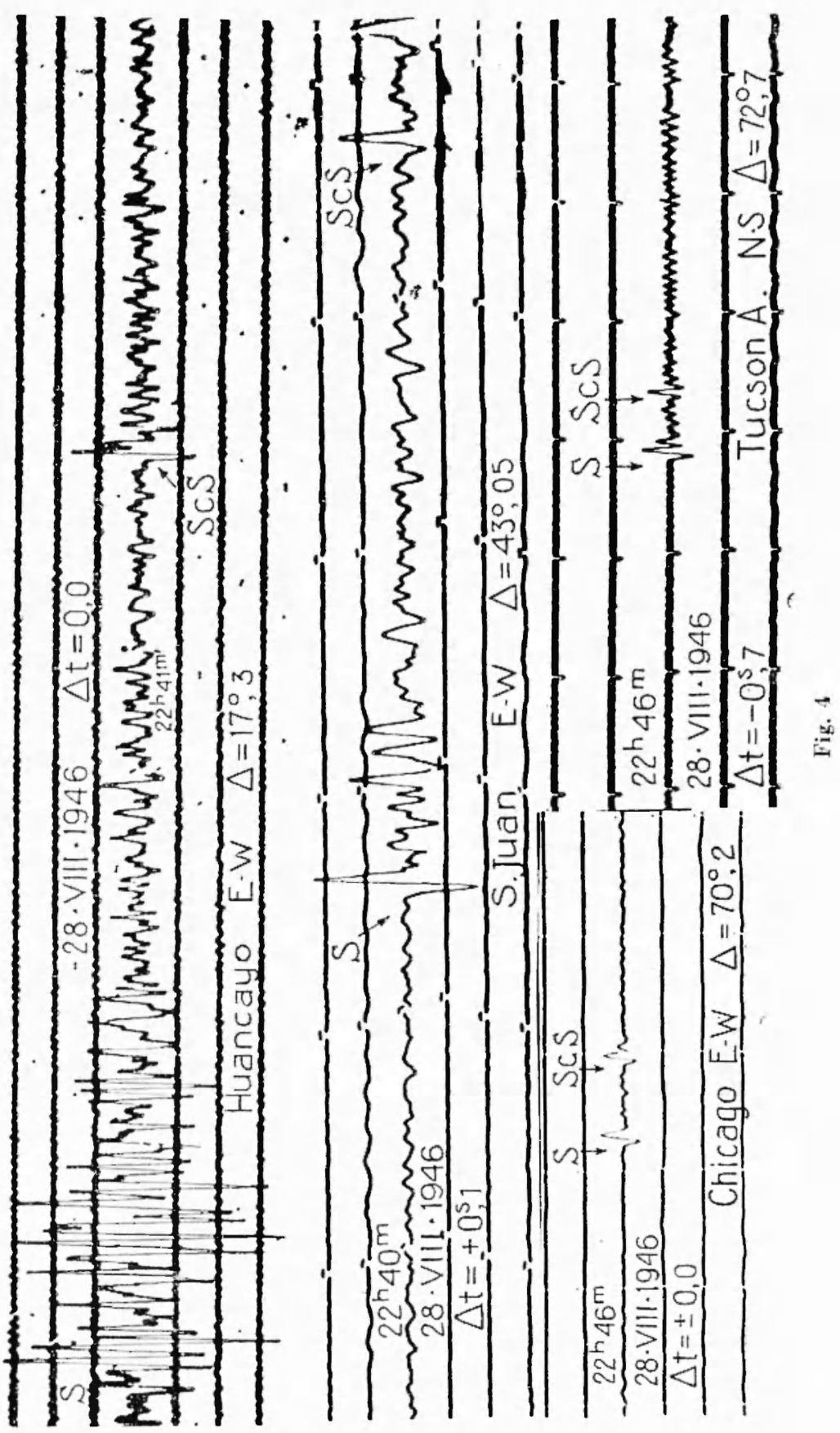


a per le varie stazioni. Poiché, in questo caso, non era possiljile valerei delle tavole calcolate da Gutenberg e Richter, si è proceduto come segue.

E noto the la distanza angolare $d \Theta$ è data dall'espressione

$$
d \Theta==\frac{k d r}{r \sqrt{n^{2} r^{2}--k^{2}}}
$$

cioè, con la simbologia già usata,

$$
d \Theta=\frac{a \bar{a} \varrho}{\varrho \sqrt{4-a^{2}}} .
$$

Per l'onda $S c S$ arremo pertanto

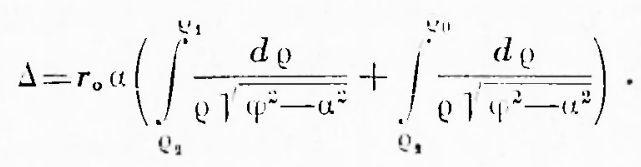

E per la [3], infine,

$$
\begin{aligned}
& \Delta=2 r_{0} u\left\{\frac{\frac{1}{a} \operatorname{arctg} \frac{\sqrt{u^{2}-a^{2}}}{a}}{a}-\frac{1}{\sqrt{\frac{c}{c}+a^{2}}} \operatorname{arctg} \frac{\sqrt{q^{2}-a^{2}}}{\sqrt{\frac{c}{c}+\omega^{2}}} \mid\right. \\
& \left(\sqrt{\varphi^{2}-a^{2}}\right)_{1} \\
& \left(\sqrt{4^{2}-u^{2}}\right)_{2} \\
& +\left|\frac{1}{\alpha} \operatorname{arctg} \frac{\sqrt{q^{2}-u^{2}}}{\alpha}-\frac{1}{\sqrt{\frac{c}{C}+\alpha^{2}}} \operatorname{arctg} \frac{\sqrt{q^{2}-u^{2}}}{\sqrt{\frac{c}{c}+\alpha^{2}}}\right| \\
& \left(\sqrt{q^{2}-\alpha^{2}}\right)_{2}
\end{aligned}
$$

Assegnati ad $\alpha$ opportuni valori, la [5] consente di determinare i corrispondenti valori di 1 . Per $u=0,07 ; 0,1 ; 0,2 ; 0,3$ si è oltenuto $\Delta=1049 ; 1515 ; 3359 ; 7722 \mathrm{~km}$ rispettiqamente. Ciò ha consentito di tracciare un grafico (fig. 3 ) da cui è possiljile trarre $i$ valori di $\alpha$ corrispondenti alle stazioni citate.

Applicando la [4] alle varie stazioni si trova allora che mentre Huancayo e S. Juan conducono entrambe ad un valore di $2920 \mathrm{~km}$ 
per la profondita del nucleo, le stazioni lontane (Clicago e Tueson) portano a scarti notevoli da retio valore.

Abbiano visto che, con la distribuzione della velocità ottenuta da Gutenberg e Richter, tutte le stazioni hanno condotto concordemente alla profondita di $2920 \mathrm{~km}$. Il fatto che con la distriluzione di Dahm corrispondano, per le stazioni lontane, notevoli scarti da detla profondita, non pui essere attribuito ad errori accidentali nei tempi registrati da quelle stazioni, bensi arl alcunché di erroneo nella distribuzione di velociti ottenuta a Dalm, che si manifesta in morlo netto solo a partire da una deteminata distanza epicentrale.

Conchulendo, i chiari esempi di onde ScS forniti dal terremoto in esame, conducono, con l'ausilio delle formule ottenute, alla conferma che la profondita del nucleo è di $2920 \mathrm{~km}$ circa, conformemente al valore ottenuto da Gutenberg, e da Gutenberge Richter, con il metodo di Wiechert-Ierglotz. Sembra pertanto che i risultati cui pervenne Dahm, con lo stesso metorlo, non rispondano a realta.

Roma - Istituto Nazionale di Geofisica - Ottobre 1949.

\section{RIASSLNTO}

Sulla scorta delle registrazioni di onde ScS, determinate dal terremoto profondo del 28 agosto 1916 , ci siamo proposti di calcolare la profondití del nucleo terrestre. Calcolati l'epicentro e la profonditi iporentrale, con un metode basato sulle equazioni fondamentali della teoria dei rasgi sismici siamo passati alla determinasione dei tempi di tragitlo delle onde SaS. A tale scopo, ci siamo valsi dei valori delle velocita di propagazione delle onde trasversali ottenuti e da Gutenberge Richter e da C. G. Dahm. Nel primo caso, si è ottenuta una protondità del nucleo pari a $2920 \mathrm{hm}$, in oltimo accordo con il valore generalmente accettato: nel secondo caso, i valori avuti risultarono alquanto discordanti. Sembra quindi che la distribuzione della velocití delle $S$ con la profondità determinata da Gutenberg e Richter sia più attendibile di quella formita da Dahm. 


\section{BIBLIOGRAFIA}

(') Caloi P. e Plinovici F.: Il terremolo del Turlestan del 2 novembre $19 \%$. Annali di Geofisica, 1, 2 (1948).

(2) Gutiniblikg B. and RuthitiR C. F.: On seismic waves, $W$. Gerlands Beitr. z. Geopliys., I.1V, pag. 102 (1939).

Kaboya K. and Hunka S.: On the ScS Wace of Deep-foctus Earthquakes observed near the Epicenter. The Geophysical Magazine, VIIl, 1 (193.).

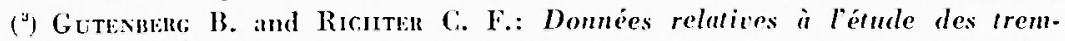
blements de terre à foyer profond. [I.G.G.I. Ass. de Sëism., Travaux scientifipues, fasc. 15, 1 partic (1937).

(') DAIn C. G.: Velocities of $P$ and $S$ ates calculated from the observed times of the Long Beach earthquake. Bull. of the Seismol. Soc. of America, XXVI, pag. 159 (1936). 